\title{
Points to consider when assessing relationships (or suspecting misattributed relationships) during family-based clinical genomic testing: a statement of the American College of Medical Genetics and Genomics (ACMG)
}

\author{
Joshua L. Deignan, $\mathrm{PhD}^{1}$, Elizabeth Chao, $\mathrm{MD}^{2}$, Jennifer L. Gannon, MD ${ }^{3,4}$, Henry T. Greely, JD ${ }^{5}$, \\ Kelly D. Farwell Hagman, MS, $\mathrm{CGC}^{6}$, Rong Mao, $\mathrm{MD}^{7}$ and \\ Scott Topper, PhD ${ }^{8}$; ACMG Laboratory Quality Assurance Committee ${ }^{9}$
}

\begin{abstract}
Disclaimer: This statement is designed primarily as an educational resource for medical geneticists and other clinicians to help them provide quality medical services. Adherence to this statement is completely voluntary and does not necessarily assure a successful medical outcome. This statement should not be considered inclusive of all proper procedures and tests or exclusive of other procedures and tests that are reasonably directed to obtaining the same results. In determining the propriety of any specific procedure or test, the clinician should apply his or her own professional judgment to the specific clinical circumstances presented by the individual patient or specimen.

Clinicians are encouraged to document the reasons for the use of a particular procedure or test, whether or not it is in conformance with this statement. Clinicians also are advised to take notice of the date this statement was adopted, and to consider other medical and scientific information that becomes available after that date. It also would be prudent to consider whether intellectual property interests may restrict the performance of certain tests and other procedures.
\end{abstract}

Keywords: parentage; sequencing; incidental/secondary finding; consent; variant
Genetics in Medicine (2020) 22:1285-1287; https://doi.org/10.1038/s41436020-0821-6

\section{INTRODUCTION}

Trio-based genetic analysis (typically involving a child and their biological parents) is an important tool in clinical diagnostic testing, as this type of analysis aids in developing an accurate understanding of the inheritance of variants observed in the proband. ${ }^{1-5}$ Understanding if a variant is inherited or is de novo can directly affect variant classification and result interpretation; consequently, misunderstanding the true biological relationship between analyzed samples can lead to erroneous clinical interpretations.

An accurate trio-based analysis cannot be completed using samples that are not biologically related. Therefore, it is recommended that laboratories performing trio analyses assess the relatedness of the submitted samples before completing the analysis.
In assessing sample relatedness, laboratories will inevitably identify cases where the submitted samples appear inconsistent with the relationships asserted on the test requisition, ${ }^{6}$ and laboratories should have policies and procedures in place which describe how to handle these cases. This "points to consider" document describes the central concepts laboratories should consider when establishing these policies and procedures.

\section{GENERAL CONSIDERATIONS}

- Trio analyses may be performed to help address questions of clinical urgency. While assessing relatedness is essential for accurate result interpretation, it is secondary to the clinical intent of the ordered test. Secondary considerations should not become obstacles to effective clinical care.

\footnotetext{
${ }^{1}$ Department of Pathology and Laboratory Medicine, University of California, Los Angeles, Los Angeles, CA, USA; ${ }^{2}$ Division of Genetics and Genomics, Department of Pediatrics, University of California Irvine School of Medicine, Irvine, CA, USA; ${ }^{3}$ Division of Clinical Genetics, Children's Mercy Hospital, Kansas City, MO, USA; ${ }^{4}$ Department of Pediatrics, University of Missouri-Kansas City, Kansas City, MO, USA; ${ }^{5}$ Center for Law and the Biosciences, Stanford Law School, Stanford, CA, USA; ${ }^{6}$ Ambry Genetics, Aliso Viejo, CA, USA; ${ }^{7}$ ARUP Laboratories, University of Utah, Salt Lake City, UT, USA; ${ }^{8}$ Color, Burlingame, CA, USA; ${ }^{9}$ American College of Medical Genetics and Genomics, Bethesda, MD, USA. Correspondence: ACMG (documents@acmg.net)

The Board of Directors of the American College of Medical Genetics and Genomics approved this statement on 16 March 2020.
} 
- The observation that submitted samples do not appear to be related is not a clinically actionable or clinically urgent observation. This observation should not be considered to be a reportable incidental/secondary finding in the same way that, for example, a pathogenic $B R C A 1$ variant might be (see the associated references for other American College of Medical Genetics and Genomics [ACMG] documents that address those issues). ${ }^{7,8}$ There is no duty to disclose this observation from a clinical framework.

- The observation that submitted samples do not appear to be related is not an actionable observation in terms of a perception of imminent harm or abuse. This observation should not be considered to be a reportable incidental/secondary finding in the same way that, for example, an observation of excessive consanguinity might be (see the associated reference for an ACMG document that addresses those issues). ${ }^{9}$ A laboratory has no duty to disclose this observation from a legal framework.

- There can be many reasons why submitted samples may not appear to be biologically related, and the test performed cannot typically distinguish among them. Laboratories are not under any legal or regulatory obligation to investigate the cause.

- One possible explanation for the observation that submitted samples do not appear to be related is misattributed parentage. While there are circumstances where that consideration can be beneficial or of interest, there are also circumstances where that subject can be sensitive and can suggest a breach of trust. The laboratory is not in a position to judge if the net value of raising this unconfirmed suspicion is helpful or harmful. Relatedness testing and carrier testing are both commercially available through direct retail venues, and if a family has an interest in those kinds of data, they are available directly using tests validated for those specific purposes.

- Patients, clinicians, and laboratories are all aligned in their pursuit of accurate test results. Sharing information that can help lead to accurate test results is in everyone's interest. If the reason a test cannot be completed is not shared, the ordering clinician cannot help address the root cause.

\section{CONSIDERATIONS FOR CONSENTING THE PATIENT (FOR THE CLINICIAN)}

The consent process should clearly explain:

- What samples are required: Trio analyses require data from three biologically related samples to be completed.
- What analyses will be performed on those samples: The data from the three samples will be compared to understand the inheritance of variants observed in the child's sample. Relatedness will be assessed on the three samples to ensure that the data is informative for this purpose.

- Reasons why the test might not be completed: If data from all three samples cannot be obtained, or if the relatedness assessment determines that one or more of the samples is not informative, the trio test will not be completed, but an alternate analysis (proband-only or duo analysis) may still be performed.

- What will be communicated in the event that the analysis cannot be completed as a trio: The clinician should clearly present to the patient(s) or guardians the laboratory's policy for communicating the reasons as to why a trio analysis could not be completed and should inform them that the laboratory may also want to attempt to obtain an additional sample from the uninformative individual(s) before proceeding with an alternate analysis.

\section{CONSIDERATIONS FOR TESTING AND INTERPRETATION (FOR THE LABORATORY)}

- Because an accurate trio analysis can only be completed on data from biologically related samples, assessing relatedness is an essential quality control measure and should be performed. It can be performed as an alternate analysis on the primary data or using data from an alternate assay, but it should be completed before results are returned.

- The degree of relatedness between two individuals is not typically provided as a standalone result or offered as a standalone test using data originating from family-based clinical genomic testing.

- Formal relationship testing is typically performed in specially licensed laboratories using short tandem repeat-based tests specifically validated for this purpose. Relatedness testing as an in-line quality control measure should not be confused with a validated test for relatedness.

- If data from three biologically related samples are not available, it may still be possible to perform a probandonly or duo analysis. The laboratory's policy about reflexing to these other analyses should be clearly stated in the test requisition and consent.

- Variants that are present in the proband and absent in both parents can only be referred to as de novo when the 
relatedness between each parent and the proband has been assessed and both are consistent with expectations. If the relatedness of one or both parents with the proband appears inconsistent with expectations, these types of variants should not be referred to as de novo.

- There are many possible explanations for an observed mismatch among submitted samples. These include accidental preanalytical sample mix-up, bone marrow transplant (hematopoietic cell engraftment), gamete donation, or a lack of biological relatedness that is known by some, all, or none of the parties. The simple observation that submitted samples are not biologically related cannot distinguish between the potential causes.

\section{CONSIDERATIONS FOR COMMUNICATION (FOR THE LABORATORY, CLINICIAN, AND GENETIC COUNSELOR)}

- Communication of the results of clinical genomic testing to the family is an important part of the practice of genomic medicine.

- Reference laboratories primarily communicate with clinicians and genetic counselors via the written report.

- If laboratories orally communicate their observation of an apparently genetically unrelated sample with the clinician or genetic counselor, details of this conversation should also be recorded in the medical record and/or laboratory information system.

- If a test cannot be completed as ordered, the laboratory and ordering clinician can be partners in troubleshooting that failure. Communicating the specific reason the test cannot be completed can aid in this troubleshooting.

\section{CONSIDERATIONS FOR REPORTING (FOR THE LABORATORY)}

- When an order is placed and a sample is processed, it is good practice for a laboratory to return a report, even if only to document that the test could not be completed. If a different test than what was ordered is performed (i.e., proband-only instead of trio), that should be documented as well.

- Clinical reports become part of the patient's medical record. Many clinicians routinely provide the family with a copy of the report, and all patients have a right to access that record per the Health Insurance Portability and Accountability Act of 1996 (HIPAA). Therefore, the patient/family perspective should be considered when writing the report.

- While diagnostic tests are typically considered to be a part of the proband's medical record, trio testing strains traditional concepts of medical record privacy. Information about both parents may appear in the child's record. Because both parents typically have rights to their child's record, each parent has indirect access to information about the other parent. Laboratories should presume that all members of the trio will have access to the proband's report.

- Because there are many possible explanations for an observed mismatch among submitted samples, and because some of those explanations can be sensitive, laboratories should avoid speculating on the report about possible causes for an observed mismatch.

\section{DISCLOSURE}

All authors except J.L.G. and H.T.G. serve as directors in clinical laboratories that perform a breadth of genetic and genomic analyses on a fee-for-service basis.

Publisher's note Springer Nature remains neutral with regard to jurisdictional claims in published maps and institutional affiliations.

\section{REFERENCES}

1. Rehm HL, Bale SJ, Bayrak-Toydemir P, et al. ACMG clinical laboratory standards for next generation sequencing. Genet Med. 2013;15:733-747.

2. Lee $H$, Deignan JL, Dorrani $N$, et al. Clinical exome sequencing for genetic identification of rare Mendelian disorders. JAMA. 2014;312:1880-1887.

3. Yang Y, Muzny DM, Reid JG, et al. Clinical whole-exome sequencing for the diagnosis of Mendelian disorders. $\mathrm{N}$ Engl J Med. 2013;369:1502-1511.

4. Retterer K, Juusola J, Cho MT, et al. Clinical application of whole-exome sequencing across clinical indications. Genet Med. 2016;18:696-704.

5. Farwell KD, Shahmirzadi L, El-Khechen D, et al. Enhanced utility of familycentered diagnostic exome sequencing with inheritance model-based analysis: results from 500 unselected families with undiagnosed genetic conditions. Genet Med. 2015;17:578-586.

6. Eno C, Bayrak-Toydemir P, Bean L, et al. Misattributed parentage as an unanticipated finding during exome/genome sequencing: current clinical laboratory practices and an opportunity for standardization. Genet Med. 2019:21:861-866

7. Green RC, Berg JS, Grody WW, et al. ACMG recommendations for reporting of incidental findings in clinical exome and genome sequencing. Genet Med. 2013;15:565-574.

8. Kalia SS, Adelman K, Bale SJ, et al. Recommendations for reporting of secondary findings in clinical exome and genome sequencing, 2016 update (ACMG SF v2.0): a policy statement of the American College of Medical Genetics and Genomics. Genet Med. 2017:19:249-255.

9. Rehder CW, David KL, Hirsch $B$, et al. Standards and guidelines for documenting suspected consanguinity as an incidental finding of genomic testing. Genet Med. 2013;15:150-152 